\title{
A Heuristic Derivation of a Planck-Heisenberg Energy Formula
}

\author{
Gregory L. Light \\ Department of Management, ProvidenceCollege \\ 1 Cunningham Square, Providence, Rhode Island02918, USA
}

Tel: 1-401-865-2662 E-mail: glight@providence.edu

\author{
Received: August 28, 2011 Accepted: September 18, 2011 Published: February 1, 2012 \\ doi:10.5539/apr.v4n1p138 \\ URL: http://dx.doi.org/10.5539/apr.v4n1p138
}

\begin{abstract}
We derive an equation of the energy of a particle equal to Planck $h$ times the frequency plus/minus the Heisenberg uncertainty energy as based on our previously constructed "combined spacetime four-manifold." At the same time, we provide more details to our spacetime geometry, in particular, that of the "wave universe."
\end{abstract}

Keywords: Conformal manifolds, Dimensional analysis, AdS/CFT, Vacuum energy, Cosmological constant, P-branes, Renormalization, Particle-wave duality

\section{Introduction}

This paper seeks to derive a single equation that explains the energy of a particle equal to that as accounted for by the Planck frequency formula plus that as accounted for by the Heisenberg uncertainty principle.The significance of the Planck formula lies in its therein contained Planck constant $h=6.62606957(29) \times$ $10^{-34} J \cdot S$ (CODATA 2010) (for a recent theoretical derivation of $h$, cf. (Brodsky \& Hover, 2011]). Historically the formula, $E=h v$, arose from Planck's derivation of the energy density $u(v)$ of the blackbody radiation in 1900 (cf. [Longair, 1984], pp. 201, 205-206 for an elaboration on the analysis in [Planck, 1901]), when Planck related $E$ (Equation (6), ibid.)to the $v$ in Wien's equation (cited by Planck as Equation (10), ibid.) through a joint expression of $u(v)$ in:

$$
u(v)_{\text {Wien }}=\frac{8 \pi h v^{3}}{c^{3}} \frac{1}{e^{(h v / k T)}}=\frac{8 \pi h v^{3}}{c^{3}} \frac{1}{e^{(E / k T)}-1}=u(v)_{\text {Planck }} .
$$

Here we can see that there is a discrepancy of the extra term of (-1) in the denominator in equating $E$ with $h v$. [Einstein, 1905] gave another derivation of $E=h v$, where he made use of the quantity (in his Section 4),

$$
\frac{1}{T}=\frac{1}{h v / k} \ln \frac{8 \pi h v^{3}}{c^{3} u(v)_{\text {Wien }}} \text { (as cast in [Longair, 1984], p.231); }
$$

here again, had Einstein used $u(v)_{\text {Planck }}$, he would not have been able to obtain the above expression of $T^{-1}$ needed for the conclusion of $E=h v$. It must be stressed however that Einstein did note the readers about the limitations of $E=h v$. ("Wirlegendiese Formelunseren Rechnungenzugrunde, behaltenaberim Sinne, dassunsere Resultatenurinnerhalbgewisser Grenzengelten." Ibid., Section 4.) Yet from our literature research the validity of this equation has never been examined (cf. e.g., [Zeidler, 2006], p. 141, for how firmly and universally this formula has been established), and additional derivations of the formula do not appear to exist (except for a recently proposed generalization by [Jou \& Mongiovi, 2011]).

Coincidentally, we encountered a similar situation in our previous article (Light, 2011, APR), where we found an extra term $\Delta \hat{E}_{j}$ added onto $h \boldsymbol{v}_{j}^{[1]}$ to account for $\hat{E}_{j}$, and this $\Delta \hat{E}_{j}$ originated from the term of 1 in our previousequation,

$$
g_{11}^{[2]}=1-\frac{2 G^{[2]} E_{j}^{[2]}}{\frac{\lambda_{j}}{2} \cdot c^{4}} \equiv 1-\frac{4 G^{[2]} E_{j}^{[2]} v_{j}^{[1]}}{c^{5}} .
$$


We had conjectured that this $\Delta \hat{E}_{j}$ was the Heisenberg's uncertainty energy (see [Samuel, 1927] for an earlier inquiry), and indeed here we will present a proof to confirm our conjecture.

In the ensuing Section 2.1, we will derive our "Planck + Heisenberg" energy formula. We note that the background of this section will be the spacetime before the Big Bang, composed purely of electromagnetic waves in a universe referred to as $\mathbf{M}^{[2]}$ in our previous article. Then in Section 2.2, we will essentially cast the recognized post-Big-Bang universein our model and derive $\Delta E=\frac{1}{2} \frac{\hbar}{\Delta t}$. Then in Section 2.3, we will prove a lemma that is actually involved in our energy formula. Finally in Section 3 we will conclude with some summary remarks.

\section{Analysis}

2.1 A single electromagnetic wave $\lambda$ by Maxwell Equations in a universe $\mathbf{M}^{[2]}$ devoid of matter

Let a reference frame $\mathrm{S}$ with a parameter domain $\left\{(t, x, y, z) \in \mathbf{R}^{1+3}\right\} \equiv \boldsymbol{\alpha}$ be given, and assume that relative to $\mathrm{S}$ there exists an electromagnetic field made up of

$$
\begin{aligned}
& \mathbf{E}=\left(0, A \cos \left(k x-\omega t-\phi_{\alpha}\right), 0\right), \\
& \langle\text { unit of } A\rangle=\frac{\text { newton }}{\text { coulomb }}, k=\frac{\omega}{c}, \phi_{\alpha} \in[0,2 \pi]
\end{aligned}
$$

varying with coordinate systems $\{\alpha\}$ byartificial spatial-temporal translations but otherwise Lorentz (geometrically) invariant with all inertial framesand

$$
\mathbf{B}=\left(0,0, \frac{A}{c} \cos \left(k x-\omega t-\phi_{\alpha}\right)\right)
$$

with the resultantPoynting vector

$$
\begin{aligned}
& \mathbf{S}=\left(\varepsilon_{0}{ }^{*} c A^{2} \cos ^{2}\left(k x-\omega t-\phi_{\alpha}\right), 0,0\right), \\
& \left\langle\text { unit of } \varepsilon_{0}{ }^{*} c A^{2}\right\rangle=\text { joule } /\left(s \cdot m^{2}\right) .
\end{aligned}
$$

Here we note that the superscript of an asterisk as attached to the permittivity constant denotes its pre-Big Bang value and that such superscript representations will consistently be adopted in the sequel, with their relationships to the present laboratory values clarified in Section 2.2.

We now solve the equation $k x-\omega t=\phi_{\alpha}$ for $x$ :

$$
\begin{aligned}
& k x-\omega t=k\left(x-\frac{\omega}{k} t\right)=\frac{2 \pi}{\lambda}(x-c t) ; \text { thus, } \\
& x=c t+\frac{\lambda}{2 \pi} \phi_{\alpha}, \forall t \in \mathbf{R}, \text { in particular, } \\
& t=0 \Rightarrow x \in[0, \lambda] .
\end{aligned}
$$

Identifying $0 \equiv \lambda$ to form a quotient space as referenced by a frame $\tilde{S}$ traveling with $[0, \lambda]$, we have $[0, \lambda] \equiv S^{1}$, a circle with radius $\frac{\lambda}{2 \pi}$, or

$$
S^{1}=\left\{(y, z) \mid y=\frac{\lambda}{2 \pi} \cos \theta, z=\frac{\lambda}{2 \pi} \sin \theta, \theta \in[0,2 \pi]\right\} .
$$

Since

$$
\max _{\theta \in[0,2 \pi]}\left(\frac{\|S\|}{c}\right)=\varepsilon_{0}^{*} A^{2}\left(\text { of unit }: \frac{\text { joule }}{m^{3}}\right),
$$

we have $\frac{1}{2} \varepsilon_{0}{ }^{*} A^{2}=$ the average energy density over $S^{1} \times[0, \lambda] \equiv Y$. Thus, $\lambda$ carries a total energy of 


$$
E=\frac{1}{2} \varepsilon_{0}^{*} A^{2} \cdot \pi\left(\frac{\lambda}{2 \pi}\right)^{2} \cdot \lambda=\frac{\lambda^{3}}{8 \pi} \varepsilon_{0}^{*} A^{2} .
$$

Since to represent $E$ as a point mass $m=\frac{E}{c^{2}}$ at any particular point $x_{0} \in[0, \lambda]+c t \forall t$ would result in $\phi_{\alpha}=\frac{2 \pi}{\lambda} x_{0} \equiv \phi_{\alpha, 0}$ and thus a loss of information of $\cos \phi_{\alpha} \forall \phi_{\alpha} \in[0,2 \pi]-\left\{\phi_{\alpha, 0}\right\}$, this point mass $m$ must move from $\theta=0$ to $2 \pi$ in a motion of

$$
(x(t), y(t), z(t))=\left(c t, \frac{\lambda}{2 \pi} \cos \omega t, \frac{\lambda}{2 \pi} \sin \omega t\right) .
$$

Note that it is here in Equation (2.7) that we pass the frequency $\omega$ as referenced by frame $\mathrm{S}$ in Equation (2.0) to frame $\tilde{S}$.Identifying $[0, \pi] \equiv[\pi, 2 \pi]$ to form a quotient space in $S^{1}$, we have $m$ move in a simple harmonic motion $\xi(t)$ that is centered at $(y, z)=\left(\frac{\lambda}{2 \pi} \cos \frac{\pi}{2}, \frac{\lambda}{2 \pi} \sin \frac{\pi}{2}\right)=\left(0, \frac{\lambda}{2 \pi}\right)$, or simply at $\theta=\frac{\pi}{2}$, with a maximum displacement to $\theta=0$ and $\pi$ by $\pm\left\|\left(0, \frac{\lambda}{2 \pi}\right)-\left(\frac{\lambda}{2 \pi}, 0\right)\right\|= \pm \sqrt{2} \cdot \frac{\lambda}{2 \pi}$, i.e.,

$$
\xi(t)=\sqrt{2} \cdot \frac{\lambda}{2 \pi} \cos \omega t,
$$

with the associated potential energy

$$
P E(\xi)=\frac{1}{2} m \omega^{2} \xi^{2}=\frac{1}{2} m \omega^{2}\left(\sqrt{2} \cdot \frac{\lambda}{2 \pi} \cos \omega t\right)^{2}=m c^{2} \cos ^{2} \omega t=E \cos ^{2} \omega t,
$$

implying in particular that the average potential energy $P E_{\text {avg }}=\frac{E}{2}$.

Yet $\frac{2 P E(\xi)}{\xi^{2}}=m \omega^{2}=m(2 \pi v)^{2}$, so that

$$
v^{2}=\frac{2 P E(\xi)}{4 \pi^{2} m \xi^{2}}
$$

Considering the fact that

$$
P E\left(\frac{\lambda}{2 \pi}\right)=\frac{1}{2} m \omega^{2} \cdot \frac{\lambda^{2}}{4 \pi^{2}}=\frac{E}{2},
$$

we have

$$
v^{2}=\frac{2 P E_{a v g}}{4 \pi^{2} m\left(\frac{\lambda}{2 \pi}\right)^{2}}=\frac{2 P E_{a v g}}{m\left(\frac{c}{v}\right)^{2}},
$$

so that

$$
P E_{\text {avg }}=\frac{1}{2}\left(\frac{m c^{2}}{v}\right) \cdot v=\left(\frac{E / 2}{v}\right) \cdot v \equiv h^{*} v .
$$

Denoting the Lorentz factor by $\gamma_{L}$, we conduct a Lorentz transformation of the above equation:

$$
\left(\gamma_{L} P E_{\text {avg }}\right)=\frac{1}{2}\left(\frac{m \gamma_{L} c^{2}}{v \gamma_{L}}\right) \cdot\left(\gamma_{L} v\right)=h^{*}\left(\gamma_{L} v\right)
$$


Thus, form-invariance is observed, and $\lambda$ carries a fixed amount of energy of $2\left(h^{*} / s\right)$. (Here we note in passing that the relativistic mass $m \gamma_{L}$ in the above Lorentz transformation (2.14) is valid only for slow moving inertial frames; for all inertial frames the relativistic mass should be $m$ divided by the smaller of the two eigenvalues of the Lorentz transformation, i.e., a replacement of $\gamma_{L}$ with the inverse of the smaller eigenvalue of the Lorentz transformation everywhere in (2.14) to result in the generally correct Lorentz transformation of the frequency v; cf. [Light, 2011, InTech].)

Now the average kinetic energy

$$
\begin{aligned}
& K E_{a v g}=P E_{a v g}=h^{*} v=\frac{1}{2} \cdot\left(\frac{2 h^{*} v}{v^{2}}\right) \cdot v^{2}=\frac{h^{*}}{v} \cdot v^{2} \\
& =\frac{h^{*} / s^{2}}{v}+\left(h^{*} v-\frac{h^{*} / s^{2}}{v}\right)=k^{*} T
\end{aligned}
$$

$=$ the heat energy in $\lambda+$ a heat converted mechanical energy that is lost for upholding the same constant oscillation frequency $\boldsymbol{v}$, where $k^{*} \equiv$ Boltzmann constant and $T \equiv$ Kelvin temperature. Thus,

$$
E=h^{*} v+\frac{h^{*} / s^{2}}{v} \equiv h^{*} v+\frac{\ddot{h}^{*}}{v} \text {, with } T=\frac{1}{k^{*}} \frac{\ddot{h}^{*}}{v} \text {. }
$$

This energy $E$ in Equation (2.16), in accordance with the moving frame $\tilde{S}$, is identical to the energy $E$ in accordance with the frame $\mathrm{S}$ of the originating Maxwell Equations by virtue of sharing the same frequency was specially noted after Equation (2.7). That is, measuring frequency linearly along the $x$-axis by the multiple of the wave length $\lambda$ is identical to wrapping $\lambda$ around a circle on the $(y, z)$-plane and counting the number of rotations. Applying the (time $x$ time) component of the metric tensor $g$ in Einstein Field Equations, we proceed to examine the gravitational effect of $E$ on the boundary of $\lambda$ in the universe $\mathbf{M}^{[2]}$, which has a large gravitational constant $G^{[2]}$ ( [Light, 2011, APR]) so that

$$
\begin{aligned}
& 0>g_{11}=1-\frac{2 G^{[2]} E}{\frac{\lambda}{2} \cdot c^{4}}=1-4 G^{[2]} \cdot \frac{\left(h^{*} v+\ddot{h}^{*} / v\right) \cdot v}{c^{5}} \\
& =1-\left(\frac{4 G^{[2]} h^{*}}{c^{5}}\right) v^{2}-\left(\frac{4 G^{[2]} \ddot{h}^{*}}{c^{5}}\right) ;
\end{aligned}
$$

since $g_{11}^{[2]}=\left(\frac{\Delta t_{0}^{[2]}}{\Delta t_{0}^{[1]}}\right)^{2}=-\boldsymbol{v}^{2} \cdot s^{2}$, we have simultaneously established:

$$
\begin{aligned}
& \text { (1) } E=h^{*} v+\frac{\ddot{h}^{*}}{v} \text {, and } \\
& \text { (2) } G^{[2]}=\frac{c^{5}}{4 \ddot{h}^{*}},
\end{aligned}
$$

i.e., an agreement between electromagnetism and gravity. (Cf. Lemma below in Subsection 2.3; here we also note that chronologically the above Equation (2.17) should be placed in the next Subsection 2.2 of the post-Big-Bang era, when the ratio of the proper time in $\mathbf{M}^{[2]}$ to the proper time in $\mathbf{M}^{[1]}$ becomes imaginary.)

\subsection{The transformation of $E$ of $\lambda$ into $\left(E^{[1]}, E^{[2]}\right)$ of a photon-wave}

Since $\lambda$ resides in aspacetimewith $g_{11}<0, \lambda$ is in a black hole $\mathbf{B}$ of $\mathbf{M}^{[2]}$ and is attracted to the center of $\mathbf{B}$ by gravity. We hypothesize that at the center, the infinite gravity transforms $\lambda$ into a particle-wave of energy $E \equiv E^{[3]}=E^{[1]}+E^{[2]}=\frac{3}{4} E^{[3]}+\frac{1}{4} E^{[3]}$ (see [Feynman, 1963], [Light, 2011, APR]) distributed over (photon $\gamma$, electromagnetic wave $\lambda$ ). In the laboratories of $\mathbf{m}^{[1]}$, however, $E^{[3]}$ is cast in the combined universe $\mathbf{m}^{[3]}$ as

$$
\hat{E}=\left[\left(\frac{3}{4}\right)^{2}+\left(\frac{1}{4}\right)^{2}\right] E^{[3]}=\frac{10}{16} E^{[3]} \text { (ibid.), }
$$


or

$$
\begin{aligned}
& E^{[3]}=1.6 \hat{E}, \text { with } \\
& E^{[1]}=1.6 \hat{E} \times \frac{3}{4}=1.2 \hat{E} \equiv \phi^{[1]} \hat{E} \text { and } \\
& E^{[2]}=1.6 \hat{E} \times \frac{1}{4}=0.4 \hat{E} \equiv \phi^{[2]} \hat{E} .
\end{aligned}
$$

Here we draw the reader's special attention to the above formulas of conversion that convert any laboratory-measured quantities (as denoted by an overhead carat) to their true quantities in $\mathbf{M}^{[i]}, i=1,2$. Thus,

$$
\begin{aligned}
& E \equiv E^{[3]}=h^{*} v+\frac{\ddot{h}^{*}}{v}=1.6\left(h v+\frac{\ddot{h}}{v}\right) \text { and } \\
& \hat{E}=h v+\frac{\ddot{h}}{v} .
\end{aligned}
$$

Now since $\frac{1}{4} E$ remains in $\mathbf{M}^{[2]}$, we have a revised (cf. Equations (2.17), (2.18))

$$
G^{[2]}=\frac{c^{5}}{4 \ddot{h}^{*} \cdot\left(\frac{1}{4}\right)}=\frac{c^{5}}{1.6 \ddot{h}} ;
$$

here (see [Light, 2011, APR]) the gravitational constant recognized in the laboratory is

For the Poynting vector, we have

$$
G^{[3]}=\frac{G^{[1]} G^{[2]}}{G^{[1]}+G^{[2]}} \approx G^{[1]} \approx 10^{-85} G^{[2]} .
$$

$$
\begin{aligned}
& \hat{\mathbf{S}}=\frac{10}{16} \mathbf{S}=\left(\frac{10}{16} \varepsilon_{0}{ }^{*} c A^{2} \cos ^{2}\left(k x-\omega t-\phi_{\alpha}\right), 0,0\right), \\
& \equiv\left(\varepsilon_{0} c A^{2} \cos ^{2}\left(k x-\omega t-\phi_{\alpha}\right), 0,0\right)=\varepsilon_{0} c^{2} \mathbf{E} \times \mathbf{B}
\end{aligned}
$$

with $\mathbf{E}$ and $\mathbf{B}$ staying the same, and $\mathbf{S}^{[2]}=\phi^{[2]} \hat{\mathbf{S}}=0.4 \hat{\mathbf{S}}$. The wavefunction is then

$$
\psi(t, x, y, z)=z_{0} \cdot\|\mathbf{E}(t, x, 0,0)\|_{\mathbf{c}^{3}}=z_{0} \cdot i \sqrt{\frac{E^{[2]}}{V}} \cos \left(k x-\omega t-\phi_{\alpha}\right),
$$

where $V \equiv \varepsilon_{0}{ }^{*} \cdot \frac{\lambda^{3}}{8 \pi}$ is from Equation (2.6), the appearance of $i$ is due to $g_{11}<0$, and $z_{0}$ is such that

$$
\int_{t_{0}}^{t_{0}+\frac{\lambda}{c}} \int_{c t}^{c t_{0}+\lambda}|\psi(t, x, 0,0)|^{2} d x d t=1 \quad \forall t_{0} \in \mathbf{R}
$$

Here, we note that as an inertial frame moves away from a light source with speed approaching c, the wave length of the light approaches infinity; therefore, a photon is to appear within any wave length $\lambda$ with probability 1 . To be complete, the probability current density is then

$$
\mathbf{j}(t, x, 0,0)=\left(\left|z_{0}\right|^{2} /\left(\varepsilon_{0} \phi^{[2]}\right)\right) \cdot \mathbf{S}^{[2]}(t, x, 0,0) .
$$

We hypothesize that any representative point mass $m$ in $[x, x+\lambda]$ of energy $E \equiv E^{[3]}$ in $\mathbf{m}^{[2]}$ may find itself immersed in $\mathbf{M}^{[1]}$ as well as $\mathbf{M}^{[2]}$, revealing itself as a photon $\gamma$ in $\mathbf{m}^{[1]}$ and that $\gamma$ emerges from $x^{*} \in[x, x+\lambda]$ into $\mathbf{M}^{[1]}$ in accordance with the probability densities $|\psi(t, x, 0,0)|^{2}$ of Equations (2.24) and (2.25). Furthermore, Equation (2.23) has an average of $0.5 \hat{\mathbf{s}}$ over $[x, x+\lambda]$. This implies that if the photon $\gamma$ carries an average energy density of $(5 / 16) \varepsilon_{0}^{*} \mathrm{~A}^{2}$ over $[x, x+\lambda]$, then it must travel ( $c$ second) in one second; however, if $\gamma$ carries an average energy density of $\varepsilon_{0}^{*} \mathrm{~A}^{2}$ over $[x, x+\lambda]$, then it only travels $[(5 / 16) c$ second $]$ in one second 
but this must mean (5/16) second. That is, the time duration of $\gamma$ in $[x, x+\lambda]$ of $\mathbf{m}^{[1]}$ is a random variable $\tau_{\gamma} \in[(5 / 16), 1](\lambda / c)$. As a result, our later derived Equation (2.32) of the Heisenberg uncertainty energy $\Delta E$ becomes an inequality: $\Delta E \geq(1 / 2)(\hbar / \Delta t)$.

Now $\gamma$ has Schwarzschild radius in the combined universe $\mathbf{m}^{[3]}$,

$$
r_{\gamma}=\frac{2 G^{[3]} \hat{E}}{c^{4}}
$$

This is a black hole in $\mathbf{m}^{[1]}$ and a white hole in $\mathbf{m}^{[2]}$, puncturing $S^{1} \times[0, \lambda] \equiv Y$ with ball $B\left(x^{*}, r_{\gamma}\right) \equiv W$. As such, $E^{[2]}$ must reside in $Y-W$. Since the Lorentz factor $\gamma_{L}$ can assume any value from 1 to infinity, there exists a reference frame relative to which the ratio

$$
\frac{\lambda}{2}: r_{\gamma}=e: 1
$$

Thus by Einstein Relativity for all inertial frames $E^{[2]}$ must be calculated

$$
\text { from } \frac{1}{e}\left(\frac{\lambda}{2}\right) \text { to }\left(\frac{\lambda}{2}\right) \text {. }
$$

To elaborate, at $r=r_{\gamma}$, time stops so that $x=c t=0$ and the entire sphere, $\left\{(x, y, z) \mid x^{2}+y^{2}+z^{2}=r_{\gamma}^{2}\right\}$, is identified with one single point in a quotient space. As such, the radius of $\lambda$ for calculating $E^{[2]}$ becomes $\left(\frac{\lambda}{2}\right)(e-1)$, a statement that will beformally established in the following Lemma.

Finally, $\left(\frac{\ddot{h}}{v}\right)=$ (the heat energy in one cycle of $\left.\lambda\left(=\frac{c \in \mathbf{R}}{v \in \mathbf{N}}\right)\right)=$ the uncertainty energy $\Delta E$ asa remainder from the division algorithm and moreover

$$
\Delta E=\frac{h}{\gamma_{L} \cdot\left(\gamma_{L}^{-1} s^{2} v\right)} \equiv \frac{h}{\gamma_{L} \cdot \widetilde{t}} \equiv \frac{h}{\Delta t} .
$$

By the symmetry in simple harmonic motions,

$$
\Delta E=\frac{1}{2} \frac{h}{\Delta t},
$$

or in terms of $\omega=2 \pi v$,

$$
\Delta E=\frac{1}{2} \frac{\hbar}{\Delta t}
$$

(cf. e.g., [Zeidler, 2009, p. 477] for the statement that harmonic oscillators achieve the Heisenberg energy lower bound, and also, [Toscano, 2006] and [Jizba, 2003] for uncertainties on small scales and examinations of the lower bounds).

\subsection{A lemma}

We prove the following Lemma:

$$
g_{11}^{[2]}:=\left(\frac{\partial t_{0}^{[2]}}{\partial t_{0}{ }^{[1]}}\right)=\left(\frac{\Delta t_{0}^{[2]}}{\Delta t_{0}^{[1]}=1 \text { second }}\right) .
$$

Proof. Let $\left(E_{j}^{[1]}, E_{j}^{[2]}\right)$ be given, where the subscript $j$ indicates the reference to a particular frame. Define

$$
z(r):=\frac{2 G^{[2]} E_{j}^{[2]}}{r c^{4}}=1-g_{11}^{[2]} .
$$


Change variable $r$ to $r=e^{s}$; then $d r=e^{s} d s$ and $\frac{d r}{r}=d s$, where s denotes the relativistically invariant multiple of $\pi$ radians and $r$ is in the unit of $\frac{\lambda_{j}}{2 e}$ (cf. [Moghimi-Araghi\& Rouhani, 2000] and [Rovelli, 1991] for spacetimeparametrizations). Then we have

$$
\int_{1}^{e} \frac{2 G^{[2]} E_{j}^{[2]}}{r c^{4}} d r \equiv \int_{0}^{1} \frac{2 G^{[2]} E_{j}^{[2]}}{c^{4}} d s=\frac{2 G^{[2]} E_{j}^{[2]}}{c^{4}}=\int_{1}^{e} z(r) d r
$$

Define the average $z(r)$ to be

$$
\begin{aligned}
& \overline{z(r)}:=\frac{\int_{1}^{e} z(r) d r}{e-1}=\frac{2 G^{[2]} E_{j}^{[2]}}{(e-1) c^{4}} \\
& \equiv 1-\overline{\left(\frac{\partial t_{0}^{[2]}}{\partial t_{0}^{[1]}}\right)^{2}} \equiv 1-\overline{\left(\frac{\partial t_{0}^{[2]}}{\partial t_{0}^{[1]}}\right)^{2}} \equiv 1-\left(\frac{\Delta t_{0}^{[2]}}{\Delta t_{0}^{[1]}}\right)^{2} .
\end{aligned}
$$

Thus, the nonlinear-terms related quantity $N L T$ in the derivative is:

$$
\begin{aligned}
& N L T=\left(\frac{\Delta t_{0}{ }^{[2]}}{\Delta t_{0}^{[1]}}\right)^{2}-g_{11}{ }^{[2]}(r) \\
& =(1-\overline{z(r)})-\left(1-\frac{4 G^{[2]} E_{j}^{[2]} v_{j}^{[1]}}{c^{5}}\right) \\
& =\frac{2 G^{[2]} E_{j}^{[2]}}{c^{4}}\left(\frac{2 v_{j}^{[1]}}{c}-\frac{1}{e-1}\right) .
\end{aligned}
$$

Since $\frac{2 v_{j}^{[1]}}{c}=\frac{1}{\left(\frac{\lambda_{j}}{2}\right)}$, we have $N L T=0$ if and only if $\frac{\lambda_{j}}{2}=e-1$, which is now derived as follows:

Let an electromagnetic wave $\Lambda$ relative to all reference frames be given. Assume that $\Lambda$ has a radius $r=1$ mmeter (where the misspelling is intentional, to mean actually "a unit of space distance") and energy $E=1$ jjoulee (again, with the intentional misspelling to mean "a unit of energy"). Denote the average energy density of $\Lambda$ over $r=1$ mmeter by $\bar{E} \equiv 1$ jjoule / mmeter. Then we have

$$
\begin{aligned}
& 1 \text { jjoule }=\int_{0 \text { mmeter }}^{1 \text { mmeter }} \bar{E} d r=\int_{0 \text { mmeter }}^{1 \text { mmeter }} 1 \cdot \frac{\text { jjoule }}{\text { mmeter }} d r=\int_{0 \text { mmeter }}^{1 \text { mmeter }} \frac{e^{r}}{e^{r}} \cdot \frac{\text { jjoule }}{\text { mmeter }} d r=\int_{r=0}^{r=1} \int_{\text {mmeter }}^{\text {mmeter }} \frac{1 \text { jjoule }}{e^{r}} \cdot d e^{r} \\
& =\int_{\widetilde{r}=1}^{\widetilde{r}=e} \frac{1 \text { jjoule }}{\widetilde{r}} d \widetilde{r}=\int_{\widetilde{r}=1}^{\widetilde{r}=e} \frac{E}{\widetilde{r}} d \widetilde{r}=\int_{k \text { meter }}^{k e \text { meter }} \frac{E}{k \widetilde{r} \text { meter }} d(k \widetilde{r} \text { meter }) \equiv \int_{\left(\lambda_{j} / 2 e\right)}^{\lambda_{j}} \frac{E_{j}(\text { of unit joule })}{R_{j}} d R_{j}
\end{aligned}
$$

relative to reference frame $j$.

Thus,

$$
R_{j} \in\left[\frac{1}{e} \frac{\lambda_{j}}{2}, \frac{\lambda_{j}}{2}\right], \widetilde{r} \in[1, e]
$$


and $\Lambda$ has a radius $\widetilde{r}=e-1$.

As such, we have provided a treatment of the pervasive problem of (1/r) (cf. a recent study by [Gralla, 2011]).

\section{Summary Remark}

In this paper we have contributed an integrated formula of the energy of a particle as based on our previous model of a combined spacetime four-manifold $\mathbf{M}^{[3]}$ :

$$
\hat{E}_{j}=h v_{j}+\frac{\ddot{h}}{v_{j}} .
$$

We thus present the above equation as a test of our model, e.g., substituting it into the Planck formula in Equation (1.0). Otherwise, we have provided additional details of the involved geometry of our model. In particular, we surmise that the ratio $(\lambda / 2 e)$ in the above Lemma may have to do with the fine-structure constant (cf. e.g., [Bouchendira, 2011] and [Tomilin, 1999]). Overall we have augmented the quantum probability setup withour combined four-dimensional spacetime geometry, and by providing a clearer temporal-spatial structure of our world we hope to facilitate a future development of quantum mechanical engineering.

\section{References}

Bouchendira, R. et al. (2011). New determination of the fine structure constant and test of the quantum electrodynamics. Physical Review Letters, 106, 080801(1-4).

Brodsky, S. J. \& Hover, P. (2011). The $\hbar$ expansion in quantum field theory.Physical Review D, 83, 045026(1-7).

Einstein, A. (1905). On a heuristic point of view about the creation and conversion of light. Annalen der Physik, 17, 132-148. http://dx.doi.org/10.1002/andp.19053220607

Feynman, R. P. et al. (1963). The Feynman lectures on physics. Reading: Addison-Wesley.

Gralla, S. E. (2011). Gauge and averaging in gravitational self-force. Physical Review D, 84, 084050(1-11).

Jizba, P. et al. (2010). Uncertainty relation on a world crystal and its applications to micro black holes. Physical Review D, 81, 084030(1-13).

Jou, D. \&Mongiovi, M. S. (2011). Duality-invariant Einstein-Planck relation and the speed of light at very short wavelengths. Physical Review D, 84, 107303(1-4).

Light, G. L. (2011). An account for dark matter and dark energy by a combined spacetime four-manifold. Applied Physics Research, 3, 130-136. http://dx.doi.org/10.5539/apr.v3n2p130

Light, G. L. (2011). General relativity extended. In A. A. Kishk (Ed.). Electromagnetic waves propagation in complex matter (pp. 157-184).Rijeka: InTech, Open Access Publisher.

Longair, M. S. (1984). Theoretical concepts in physics. Cambridge: CambridgeUniversity Press.

Moghimi-Araghi, S. \& Rouhani, S. (2000). Logarithmic conformal field theories near a boundary. Letters in Mathematical Physics,53, 49-57. http://dx.doi.org/10.1023/A:1026519016890

Planck, M. (1901). On the law of distribution of energy in the normal spectrum. Annalen der Physik, 4, 553-566. http://dx.doi.org/10.1002/andp.19013090310

Rovelli, C. (1991). Time in quantum gravity: a hypothesis.Physical Review D, 43, 442-456. http://dx.doi.org/10.1103/PhysRevD.43.442

Samuel, J. (1927). Note on the fluctuations in black body radiation, Physical Review, 30, 944-947. http://dx.doi.org/10.1103/PhysRev.30.944

Tomilin, K. A. (1999). Fine-structure constant and dimension analysis. European Journal of Physics, 20, L39-L40.http://dx.doi.org/10.1088/0143-0807/20/5/404

Toscano, F. (2006). Sub-Planck phase-space structures and Heisenberg-limited measurements, Physical Review $A, 73,023803(1-7)$.

Zeidler, E. (2006). Quantum Field Theory I: Basics in Mathematics and Physics. Berlin: Springer. http://dx.doi.org/10.1007/978-3-540-34764-4

Zeidler, E. (2009). Quantum Field Theory II: Quantum Electrodynamics.Berlin: Springer. 